\title{
Decentralised optimal deployment of mobile underwater sensors for covering layers of the ocean
}

\author{
Valimohammad Nazarzehi, Rasoul Damani
}

Maritime Engineering College, Chabahar Maritime University, Iran

\begin{abstract}
Article Info
Article history:

Received Aug 6, 2021

Revised Nov 12, 2021

Accepted Dec 8, 2021

\section{Keywords:}

Decentralised control

Mobile sensor networks

Sensing coverage

Underwater sensor networks

ABSTRACT

This paper presents the problem of sensing coverage of layers of the ocean in three dimensional underwater environments. We propose distributed control laws to drive mobile underwater sensors to optimally cover a given confined layer of the ocean. By applying this algorithm at first the mobile underwater sensors adjust their depth to the specified depth. Then, they make a triangular grid across a given area. Afterwards, they randomly move to spread across the given grid. These control laws only rely on local information also they are easily implemented and computationally effective as they use some easy consensus rules. The feature of exchanging information just among neighbouring mobile sensors keeps the information exchange minimum in the whole networks and makes this algorithm practicable option for undersea. The efficiency of the presented control laws is confirmed via mathematical proof and numerical simulations.
\end{abstract}

This is an open access article under the CC BY-SA license.

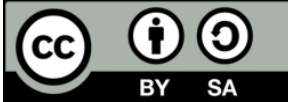

\section{Corresponding Author:}

Valimohammad Nazarzehihad

Maritime Engineering College, Chabahar Maritime University

Chabahar, Iran

Email: v.nazarzehi@cmu.ac.ir

\section{INTRODUCTION}

To improve our knowledge about ecosystems of the ocean, its dynamic, impact on the global environment and for collecting data to understand the full water environment, we require to gather spatiotemporal information not only on the surface but also at all layers of the ocean. To do this task, we need numerous sensors with capability of transmitting the collected data to the surface, which are challenging tasks in three dimensional undersea environments [1], [2]. Most of the conventional frameworks for water body dynamic study uses static sensor buoys which have limitation on their movement also they have high recovery and replacement cost. Furthermore, for many cases the deployment area is not accessible because of hostile environments or existence of mines as a result for a such case deploying static sensors may not be possible [3]. To tackle these limitations, in this paper, we take advantage of mobile underwater sensors which can be used in seabed exploration, marine monitoring, target tracking and marine military. Also, they help human to have access and manage marine information in hostile environments. A group of autonomous underwater vehicles form a mobile underwater sensor network [4]. Unlike static underwater sensor network, autonomously operated underwater vehicles have long deployment time also they can sense large regions of the ocean because they can move in all directions [5]. Besides, by using such network we can reduce the cost of operation by covering a large area with limited hardware [6]-[8]. Most of the previous sensing coverage works assumed that the mobile sensors are dispersed in a two-dimensional workspace and the sensing area, and the mobile sensors are in the same plane. This 2-D assumption is acceptable for sensing coverage on the earth surface. It is obvious that this may no longer be valid over a three-dimensional underwater space where the mobile sensors are dispersed over a three-dimensional environment [9]. Barr et al. [10] proposed a 
centralized solution to assign sensors to grid positions in 3D underwater environments. However, because of severe detection and communication constraints and massive information exchange among sensors, the centralized control is not a feasible solution in underwater environments [11]. Barr et al. [12], proposed a distributed and localized method for re-adjustment of mobile underwater sensors was presented. However, by using that technique, the mobile underwater sensors could only move up and down to modify depth for maximizing coverage. Communication bandwidth is severely limited in underwater environment [11], [13]. Therefore, designing large-scale control systems in the ocean for cooperating of autonomous mobile sensors is difficult [14]. To tackle this problem, we present a decentralized control framework which is able to minimize the information exchange among mobile underwater sensors. This algorithm relies on information from the other mobile underwater sensors located in the neighbourhood of each mobile underwater sensor. Besides, the proposed algorithm is computationally effective and simply implemented as it uses some simple local control laws [8]. Here, we focus on the problem spatiotemporal coverage of desired phenomena in a specific depth in a 3D underwater environment. In [15], a decentralised control law for full coverage of a confined two-dimensional region by a mobile underwater network was studied. The proposed algorithm was based on a 2-D workspace assumption. In this paper, the decentralized control laws introduced in [15] are developed for sensing coverage in three dimensional underwater spaces. Based on our method, to relocate the mobile underwater sensors in the same plane with the sensing area after initial deployment, firstly the underwater sensors travel toward the given depth using pressure sensors for the depth adjustment. Then, by applying the presented algorithm the mobile sensors form a triangular grid and randomly move to spread across the vertices of the grid. By using this triangular grid, we need the minimum number of mobile underwater sensors to cover the bounded given depth. To summarise, based on this decentralised random control algorithm, a given layer of the ocean can be covered by the minimum number of mobile underwater sensors. This algorithm can be used for surveying the sea floor for hydrocarbon exploration [16], [17], deep ocean floors cites monitoring [18], sensing biological and chemical phenomena in different depths [19] and equipment monitoring and leak detection [20].

\section{PROBLEM STATEMENT}

Covering a certain layer (depth) of the ocean by the minimum number of mobile underwater sensor is the main problem in this research. Here, we introduce a decentralised algorithm for deployment of a network of underwater mobile robots to cover a given bounded depth in underwater environments by least number of underwater sensors to gather scientific data from different depths and for better understanding of the ocean. The presented method consists of three stages. Initially, the mobile sensors move toward the given depth. At the end of this step all mobile underwater sensors will be in the same depth. Then, they form a triangular grid across the specified depth that covers the whole area. Finally, to optimally spread the mobile underwater sensors across the given depth, the mobile underwater sensors randomly move to occupy the vertices of the common grid. Figure 1 shows the two-dimensional bounded sensing region $\mathcal{R}$. Problem assumptions are,

$$
\begin{array}{ll}
n & : \text { number of mobile underwater sensors } \\
p_{i}(.) \in \mathcal{R}^{3} & : \text { coordinate of the mobile underwater sensor } i \\
r_{s}>0, r_{c} \geq \sqrt{3} r_{s} & : \text { mobile underwater sensors' sensing and communication range, respectively } \\
\mathcal{T}>0: & : \text { discrete time instance used for communication by mobile underwater sensors [21] }
\end{array}
$$

- Sphere-based sensing model: It is assumed each mobile underwater sensors' sensing range is $r_{s}$. It means each mobile underwater sensors can reliably sense objects within a distance of $r_{s}$ from the mobile underwater sensor's position. In the following, we define a sphere such that the mobile underwater sensor located in its center can sense objects within the sphere,

$$
\mathcal{S}_{i, r_{s}}=\left\{p \in \mathcal{R}^{3} ;\left\|\left(p-p_{i}(\mathcal{T})\right)\right\| \leq r_{s}\right\}
$$

- Sphere-based communication model: It is assumed each mobile underwater sensors has a communication range of $r_{c}>0$ will be able to exchange information with other mobile underwater sensors that are placed inside a sphere with radius $r_{c}$ centered at the mobile underwater sensors' position.

$$
\mathcal{S}_{i, r_{c}}=\left\{p \in \mathcal{R}^{3} ;\left\|\left(p-p_{i}(\mathcal{T})\right)\right\| \leq r_{c}\right\}
$$

In this paper, it is assumed that all mobile underwater sensors have the same $r_{c}$ and $r_{s}$. Moreover, it is assumed that the depth and the boundary of the sensing region are available for all mobile sensors a priori. 


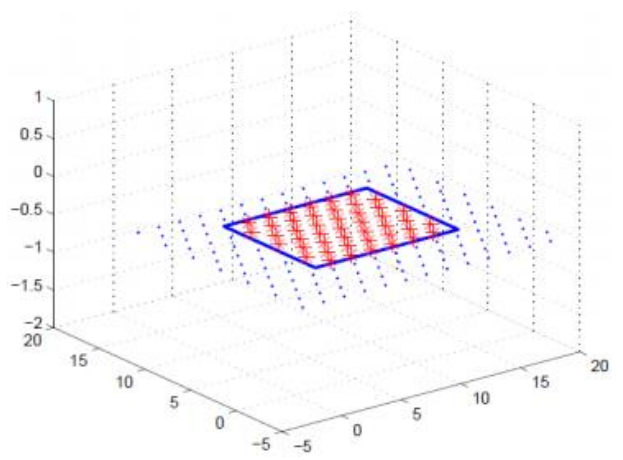

Figure 1 . The given bounded area denoted by Rectangular, $\mathcal{V}$ represented by, and $\hat{\mathcal{V}}$ represented by $*$

- Assumption 2.1: The sensing region is a bounded and connected set.

- Definition 2.3: (Triangular full coverage): suppose $\mathcal{V}$ be the unlimited set of potential triangular grids vertices that cutting the sensing region $(\mathcal{R})$ to equilateral triangles that their sides are $\sqrt{3} r_{s}$. The finite set of $\hat{\mathcal{V}}=\mathcal{V} \cap \mathcal{R}$ is said to triangular full coverage if,

$$
\forall p \in R, \quad \exists \quad v \in \widehat{V},\|(p-v)\| \leq r_{s}
$$

notice that, some part of boundary of $\mathcal{R}$ may be not covered, as shown in Figure 1. Based on the definition 2.3, the set is a triangular full coverage of the sensing region $\mathcal{R}$. As a result, if we deploy the mobile underwater sensor network and place them at vertices of the mentioned grid, then the mobile sensor network can sense any object located in the given layer of the ocean.

- Theorem 2.1: Suppose $\mathcal{R} \subseteq \mathcal{R}^{2}$ be the sensing region and the area of $\mathcal{R}$ is denoted by $\mathcal{A}(\mathcal{R})$. Suppose $\hat{\mathcal{V}}$ consist of a set of vertices of the triangular grid that cover the given layer of the ocean. Furthermore, assume that the minimum number of points we need to cover $\mathcal{R}$ is $\mathcal{N}\left(r_{s}\right)$ also suppose that the least number of vertices in $\hat{\mathcal{V}}$ is $m\left(r_{s}\right)$. Then,

$$
\lim _{r_{s} \rightarrow 0} r_{s}^{2} m\left(r_{s}\right)=\lim _{r_{s} \rightarrow 0} r_{s}^{2} N\left(r_{s}\right)=\frac{2 \sqrt{3}}{9} \mathcal{A}(\mathcal{R})
$$

see [22] and [23] for the proof.

We take advantage of the concept of graph to explain that the mobile underwater sensors communicate to other mobile sensors located in their communication range. It is obvious that the mobile underwater sensors can not exchange data to other mobile sensors out of their communication range as in underwater environments the communication range is severely limited. As a result, they should move close to each other to be able to communicate and coordinate their motion. We assume that the mobile underwater sensors form a graph $G$ so that the mobile underwater sensors are graphs' nodes and the distance between two mobile sensors is an edge of the graph. We assume that $\mathcal{N}_{i}(k)$ be the set of all mobile underwater sensors. It is obvious that two vertices of $G$ are connected if two mobile underwater sensors located on those vertices are in their communication range.

- Assumption 2.2: during time intervals $[\mathrm{km}, \mathrm{km}+1)$ which are sequence of continues, bounded and non empty, where $m=0,1,2, \ldots, G(k)$ is connected across each [km, $k m+1$ ) [22], [24], [25].

\section{DECENTRALISED COVERAGE ALGORITHM}

Sensing or sampling coverage of phenomena in a given layer of the ocean is important for gathering scientific data. Therefore, without loss of generality, let $Z=Z_{0}$ be the horizontal plane (specific depth) such that monitoring region $\mathcal{R} \subseteq Z$. We assume that the depth and the boundary of the sensing area are known for all sensors a priori. In the following, we propose a three phases algorithm for deploying a network of mobile underwater sensors to monitor a specific confined layer of ocean.

\subsection{Moving sensors to the given area}

Initially, the mobile underwater robots begin in a random distribution over the given area. We suppose that they know the depth of the monitoring region. Therefore, at the first stage they move from their initial random positions to the same depth with the monitoring region. We note that in underwater environment, $\mathcal{Z}$ (depth) can be measured precisely using pressure sensor in addition with the current 
technology there are several methods to adjust the depth of mobile underwater sensors. After adjustment of the depth of the mobile underwater sensors all of them are located at the same plane with the monitoring region.

\subsection{Forming a common grid}

Let $\Psi$ be the heading angle. To cover a specified depth (a horizontal plane), we suppose that, for any heading angle, The Vectors $n_{h_{1}}\left(\Psi ;_{0}\right)=\left(\cos (\Psi), \sin (\Psi), Z_{0}\right), n_{h_{2}}\left(\Psi ; Z_{0}\right)=\left(\cos \left(\Psi+\frac{\pi}{3}\right), \sin \left(\Psi+\frac{\pi}{3}\right), Z_{0}\right)$ and $n_{h_{3}}\left(\Psi ; Z_{0}\right)=\left(\cos \left(\Psi-\frac{\pi}{3}\right) ; \sin \left(\Psi-\frac{\pi}{3}\right) ; Z_{0}\right)$ represent the directions of three lines of the grid of the given depth $\left(Z_{0}\right)$. We use a point and an angle to define a triangular grid. Assume that $\mathcal{U}$ be a point that denotes a vertex of the grid and $\Psi_{i}(\mathcal{T})$ be an angle that represents the grid's direction respect to $\$ 1$ verb"X"\$ axis. Any angle $\Psi_{i}(\mathcal{T})$ and a point $\mathcal{U}$ form a set named $\hat{\mathcal{V}}[\mathcal{U} ; \Psi]$ that is unique. In addition, variables $\Psi_{i}(\mathcal{T})$ as well as $\mathcal{U}_{i}(\mathcal{T})$ are used as consensus variables for each mobile underwater sensor $i$ to express their heading and position, respectively. Moreover, three-dimensional vector $\mathcal{U}_{i}(\mathcal{T})=\left[\mathcal{X}_{i}(\mathcal{T}), \mathcal{Y}_{i}(\mathcal{T}), Z_{i}(\mathcal{T})\right]$ is used to represents the coordinates of the vertices of the grid, also the grids' direction is denoted by scalar consensus variable $\Psi_{i}(\mathcal{T})$.

Assumption 3.1: suppose that the initial values of the coordination variables $\left(\Psi_{0}\right)$ of the mobile underwater sensors is between 0 and $\pi$. Assume that the closest vertices of the triangular grid $\hat{v}[\mathcal{U}, \Psi]$ to mobile underwater sensors position $\mathcal{P}$ is denoted by $\mathcal{C}[\mathcal{U}, \Psi](\mathcal{P})$. Mobile underwater sensors use rules (5) and (6), to update their coordinates as well as the consensus variables,

$$
\begin{aligned}
& \Psi_{i}(\mathcal{T}+1)=\frac{\Psi_{i}(\mathcal{T})+\sum_{j \in N_{j}(\mathcal{T})} \Psi_{j}(\mathcal{T})}{1+\left|N_{j}(\mathcal{T})\right|} \\
& \mathcal{U}_{i}(\mathcal{T}+1)=\frac{u_{i}(\mathcal{T})+\sum_{j \in N_{i}(\mathcal{T})} u_{j}(\mathcal{T})}{1+\left|N_{j}(\mathcal{T})\right|} \\
& \mathcal{P}_{i}(\mathcal{T}+1)=\mathcal{C}\left[\mathcal{U}_{i}(\mathcal{T}), \Psi_{s}\right]\left(\mathcal{P}_{i}(\mathcal{T})\right)
\end{aligned}
$$

as shown, the mobile underwater sensor network uses the aforementioned rules (5), (6) to update the mobile sensors consensus variables. To update the consents variable, the mobile underwater sensors, use the average of other mobile sensors located in its communication range (neighborhood) and it own. Also, by using these rules the mobile underwater sensors achieve consensus on a point on triangular grid and the heading of the grid. After a few iterations the mobile sensor network build a common grid and then the mobile underwater sensors move toward vertices of the grid.

Theorem 3.1: assume a mobile sensor network use control laws (5), (6) to set their movement, suppose assumptions 3.1, 2.2 and 2.1 hold. Then, for all mobile underwater robots there exists a set of vertices named $\hat{v}$ such that,

$$
\lim _{k \rightarrow \infty} p_{i}(k)=v
$$

where, the location of $i_{t h}$ mobile underwater sensors is represented by $p_{i}(k)$. Also, $v$ is a vertice of the grid belongs to $\hat{\mathcal{V}}$. By using the proposed control laws, initially, the mobile underwater sensors use rule (5) to form a common grid as after applying this law the consensus values converge to constant values. Next stage, by using the control law (6) mobile underwater robots move to the vertices of the common grid (see proof in [15], [24]).

\section{FULL COVERAGE}

In the previous part, using the laws (5), (6), the whole mobile underwater robots are derived to the vertices of the common triangular lattice. However, by applying this stage the whole vertices are not occupied. For spreading the mobile underwater sensors in the whole sensing area, we propose a random algorithm. Let $S\left(p_{i}(k)\right)$ be denoted as the set that has a vertices named $\mathcal{V}$ which contain a robot that its position is repressed by $p_{i}(k)$ as well as its vacant neighbours belong to $\hat{\mathcal{V}}$. In addition, suppose that number of elements in $S\left(p_{i}(k)\right.$ is represented by $\left.\left\|S\left(p_{i}(k)\right)\right\|\right)$, which is a number between 1 and 7 . The mobile underwater sensor network takes advantage of the following random algorithm to cover the entire unoccupied vertices of the triangular grid,

$$
p_{i}(\mathcal{T})=s
$$

with probability $\frac{1}{\| S\left(p_{i}(k) \|\right.}, \forall s \in S\left(p_{i}(k)\right)$. Based on random algorithm (8), unoccupied vertices of $\hat{\mathcal{V}}$ are covered if the number of vertices in $\hat{\mathcal{V}}$ be less than the number of mobile underwater sensors. 
- Theorem 3.2: Suppose the grid $\hat{\mathcal{V}}$ has $N_{m}$ vertices also assume the mobile sensor network consists of $\mathcal{N}$ mobile underwater robots. Assume that $\mathcal{N} \geq N_{m}$ and the mobile underwater sensors spread through the sensing region based on the random law (8). Then, after a certain time $\mathcal{T} \geq \mathcal{T}_{0}$ with probability 1 for all $v \in \hat{V}$ the relationship $v=p_{i}(k)$ holds. Based on the aforementioned algorithm after a while, with probability 1 there will be a mobile underwater sensor in each vertices of the common grid. (See [21], [25] and references therein for proof.

\section{RESULTS AND DISCUSSION}

Using MATLAB software, we perform simulation to evaluate the performance of the presented control laws. We assume that there are a team of mobile underwater sensors. This network will be deployed to obtain sensing coverage within the given bounded area defined by $Z=-5$ as sensing region's depth and $1 \leq x \leq 9$ and $3 \leq y \leq 17$ are considered as its boundary, Figure 2(a). Evolution of the mobile underwater robots' position over time is shown in Figure 2. As shown in Figure 2, for the initial configuration, we randomly spread 50 mobile underwater sensors around an area of interest. Based on our proposed algorithm, initially, the underwater robots travel into the sensing area. By adjusting their depth as presented in Figure 2(b). After applying depth adjustment, all mobile underwater sensors are at the same plane with the monitoring region. To make a triangular covering grid the mobile underwater sensors take advantage of control rules (5), (6) and start moving to make the grid. Figure 2(c) shows the result of movement of the mobile underwater robots to occupy the vertices of the triangular grid.

Afterwards, random control rule (8) is used to distribute mobile underwater robots for full sensing coverage of the given depth. As shown in Figure 2(d), the mobile underwater sensors are spreading randomly in the specified layer of the ocean after 5 steps. The mobile underwater sensors completely cover the given area after 25 steps, see Figure 3.

In the following, the performance of triangular grid-based coverage is compared with square based grid. Figure 4(a) shows that the number of mobile underwater robots needed to cover the rectangular sensing area is about 100, while as shown in Figure 4(b), if we use square grid to cover this area, we need about 105 sensors due to the bigger overlapping area of the square grid. As a result, the square grid needs the more mobile underwater robots for covering the given sensing region.

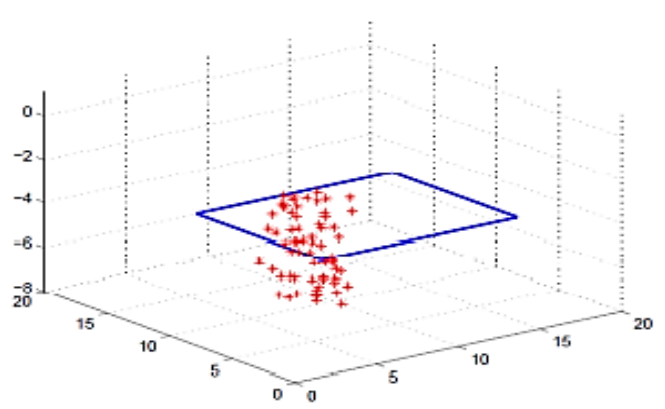

(a)

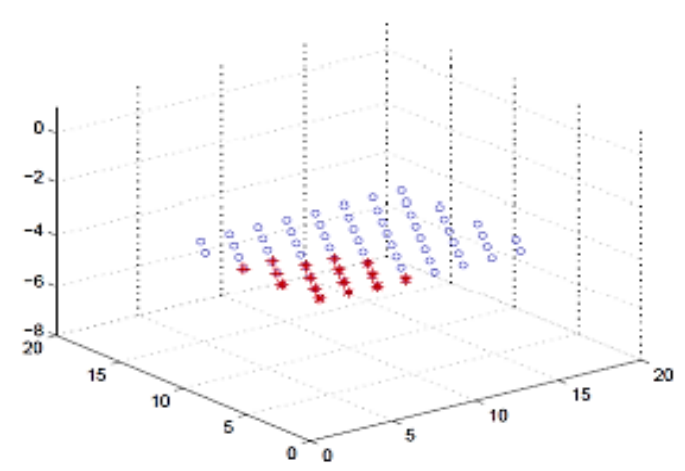

(c)

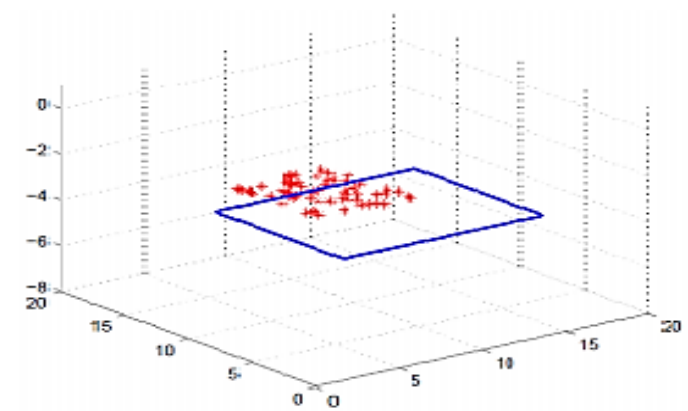

(b)

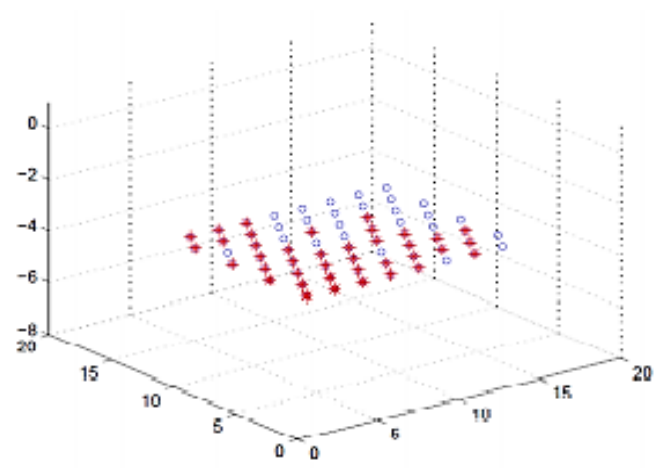

(d)

Figure 2. Mobile underwater sensors represented by *, the given area by rectangular, vertices of the grid by: (a) mobile underwater sensors' initial position, (b) sensors movement to the same plane with the monitoring region by depth adjustment, (c) applying control rules (5), (6), and (d) applying random rule (8), after $\mathcal{T}=5$ 


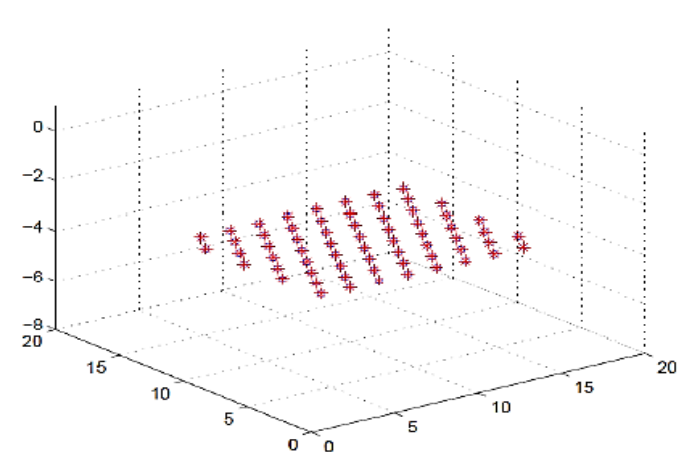

Figure 3. Complete coverage after 25 steps

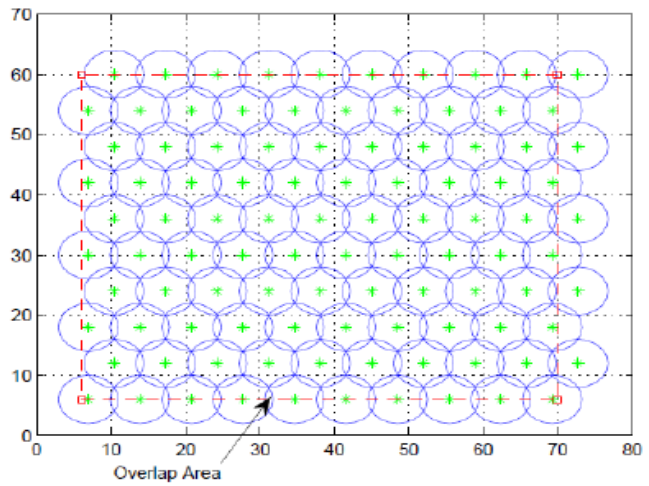

(a)

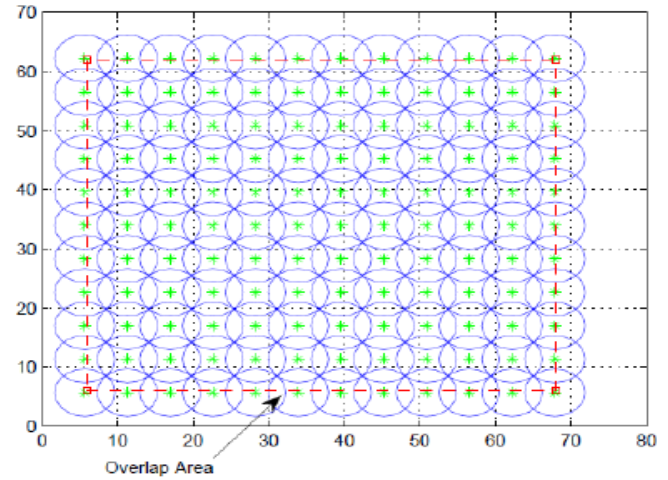

(b)

Figure 4. Comparison of the number of sensors and overlap among them between: (a) triangular-grid figure and (b) square-grid

\section{CONCLUSION}

This paper suggested some control rules for the sensing coverage of a desired layer of the ocean in three-dimensional underwater environments. After initial random deployment, by depth adjustment the mobile underwater sensors move to the same plane with the given bounded sensing region. Then, the mobile underwater sensor forms a triangular grid that covers the whole given two-dimensional area based on some simple consensus rules that are computationally effective and simply implemented. Triangular grid is better than a square grid in term of the number of mobile underwater sensors needs to cover the same are. It was shown that by using the triangular grid, we need the minimum number of mobile underwater sensors to cover the whole area. The proposed control algorithm is distributed as the mobile sensors coordinate their motion by communicating with other mobile underwater robots located in their neighborhood. Significant feature of the developed algorithm is that; relatively small amount of information exchange is required between mobile underwater sensors. Hence, this algorithm is a suitable option for communication and motion coordination in underwater environments. Performance of these presented control laws are confirmed mathematically and by computer simulations.

\section{REFERENCES}

[1] K. S. Adu-Manu, F. A. Katsriku, J.-D. Abdulai, and F. Engmann, "Smart river monitoring using wireless sensor networks," Wireless Communications and Mobile Computing, vol. 2020, 2020, doi: 10.1155/2020/8897126.

[2] C. Detweiler et al., "Adaptive decentralized control of mobile underwater sensor networks and robots for modeling underwater phenomen," Journal of Sensor and Actuator Networks, vol. 3, no. 2, pp. 113-149, 2014, doi: 10.3390/jsan3020113.

[3] V. Nazarzehi and A. V. Savkin, "Decentralized three dimensional formation building algorithms for a team of nonholonomic mobile agents," Int. J. of Control, Automation and Syst., vol. 17, no. 5, pp. 1283-1292, 2019, doi: 10.1007/s12555-018-0283-7.

[4] K. W. Al-Ani, A. S. Abdalkafor, and A. M. Nassar, "An overview of wireless sensor network and its applications," Indonesian Journal of Electrical Engineering and Computer Science (IJEECS), vol. 17, no. 3, pp. 1480-1486, 2019, doi: 10.11591/ijeecs.v17.i3.pp1480-1486.

[5] M. J. Al-Amery and M. H. Ghadban, "An energy consumption minimization approach in wireless sensor networks," Indonesian Journal of Electrical Engineering and Computer Science (IJEECS), vol. 22, no. 3, pp. 1485-1494, 2021, doi: 10.11591/ijeecs.v22.i3.pp1485-1494. 
[6] R. Priyadarshi, B. Gupta, and A. Anurag, "Deployment techniques in wireless sensor networks: a survey, classification, challenges, and future research issues," The Journal of Supercomputing, pp. 1-41, 2020, doi: 10.1007/s11227-020-03166-5.

[7] V. Nazarzehi and A. V. Savkin, "Decentralized control of mobile three-dimensional sensor networks for complete coverage selfdeployment and forming specific shapes," 2015 IEEE Conf. on Cont. App., 2015, pp. 127-132, doi: 10.1109/CCA.2015.7320621.

[8] V. Nazarzehi, A. V. Savkin, and A. Baranzadeh, "Distributed 3d dynamic search coverage for mobile wireless sensor networks," IEEE Communications Letters, vol. 19, no. 4, pp. 633-636, 2015, doi: 10.1109/LCOMM.2015.2398867.

[9] W. Wu, Z. Zhang, W. Lee, and D.-Z. Du, "Optimal Coverage in Wireless Sensor Networks," Springer, 2020, doi: 10.1007/978-3030-52824-9.

[10] S. Barr, B. Liu, and J. Wang, "Underwater sensor barriers with auction algorithms," in Proceedings of 18th International Conference on Computer Communications and Networks. IEEE, 2009, pp. 1-6, doi: 10.1109/ICCCN.2009.5235296.

[11] Y. Yang, Y. Xiao, and T. Li, "A survey of autonomous underwater vehicle formation: Performance, formation control, and communication capability," IEEE Communications Surveys \& Tutorials, vol. 23, no. 2, pp. 815-841, 2021, doi: 10.1109/COMST.2021.3059998.

[12] S. J. Barr, J. Wang, and B. Liu, "An efficient method for constructing underwater sensor barriers," Journal of Communications, vol. 6 , no. 5, pp. 370-383, 2011, doi: $10.4304 / \mathrm{jcm} .6 .5 .370-383$

[13] M. Aljanabi, M. H. Enad, and J. M. Jasim, "Study of impact of art performance level of blue laser technology applications and its control," Indonesian Journal of Electrical Engineering and Computer Science (IJEECS), vol. 5, no. 3, pp. 401-408, 2017, doi: 10.11591/ijeecs.v17.i3.pp1383-1389.

[14] G. Godi, M. R. Bharamagoudra, and Sunilkumar S. Manvi, "Survey on simulators for underwater sensor networks," Turkish Journal of Computer and Mathematics Education, vol. 12, no. 13, pp. 1719-1728, 2021, doi: 10.5296/npa.v8i4.10471.

[15] A. V. Savkin, F. Javed, and A. S. Matveev, "Optimal distributed blanket coverage self-deployment of mobile wireless sensor networks," IEEE Communications Letters, vol. 16, no. 6, pp. 949-951, 2012, doi: 10.1109/LCOMM.2012.040912.110932.

[16] J.-H. Wang, X.-B. Li, S.-J. Liu, and M.-T. Jia, "A new development direction of deep sea exploration vessels," in The 31st International Ocean and Polar Engineering Conference. OnePetro, 2021.

[17] A. M. Hasan and S. M. Rafaat, "Optimized formation control of multi-agent system using pso algorithm," Indonesian Journal of Elec. Eng. and Comp. Science (IJEECS), vol. 20, no. 3, pp. 1591-1600, 2020, doi: 10.11591/ijeecs.v20.i3.pp1591-1600.

[18] H. Mansor, M. H. Norhisam, Z. Z. Abidin, and T. S. Gunawan, "Autonomous surface vessel for search and rescue operation," Bulletin of Electrical Engineering and Informatics (BEEI), vol. 10, no. 3, pp. 1701-1708, 2021, doi: 10.11591/eei.v10i3.2599.

[19] G. S. Sosa, J. S. Abril, J. Sosa, J.-A. M.-Nelson, and T. Bautista, "Design of a practical underwater sensor network for offshore fish farm cages," Sensors, vol. 20, no. 16, p. 4459, 2020, doi: 10.3390/s20164459.

[20] S. Fattah, A. Gani, I. Ahmedy, M. Y. I. Idris, and I. A. T. Hashem, "A survey on underwater wireless sensor networks: Requirements, taxonomy, recent advances, and open research challenges," Sensors, vol. 20, no. 18, p. 5393, 2020, doi: $10.3390 / \mathrm{s} 20185393$

[21] A. V. Savkin and F. Javed, "A method for decentralized self-deployment of a mobile sensor network with given regular geometric patterns," in 2011 Seventh International Conference on Intelligent Sensors, Sensor Networks and Information Processing. IEEE, 2011, pp. 371-376, doi: 10.1109/ISSNIP.2011.6146612.

[22] A. Jadbabaie, J. Lin, and A. S. Morse, "Coordination of groups of mobile autonomous agents using nearest neighbor rules," IEEE Transactions on automatic control, vol. 48, no. 6, pp. 988-1001, 2003, doi: 10.1109/TAC.2003.812781.

[23] R. Kershner, "The number of circles covering a set," American Journal of Mathematics, vol. 61, no. 3, pp. 665-671, 1939.

[24] M. Cao, D. A. Spielman, and A. S. Morse, "A lower bound on convergence of a distributed network consensus algorithm," in Proceedings of the 44th IEEE Conference on Decision and Control. IEEE, 2005, pp. 2356-2361, doi: 10.1109/CDC.2005.1582514.

[25] N. Kottege and U. R. Zimmer, "Underwater acoustic localization for small submersibles," Journal of Field Robotics, vol. 28, no. 1 , pp. 40-69, 2011, doi: 10.1002/rob.20378.

\section{BIOGRAPHIES OF AUTHORS}

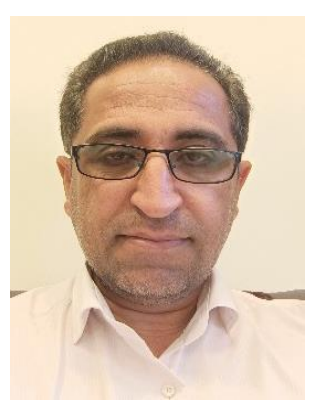

Valimohammad Nazarzehi (D) SC P received his Ph.D. degree in 2016 from the University of New South Wales, Australia. Currently, he is an assistant professor in the department of Electrical Engineering, Chabahr Maritime University, Iran. His research interests include decentralized control, marine control systems, and control of mobile robots. He can be contacted at email: v.nazarzehi@cmu.ac.ir.

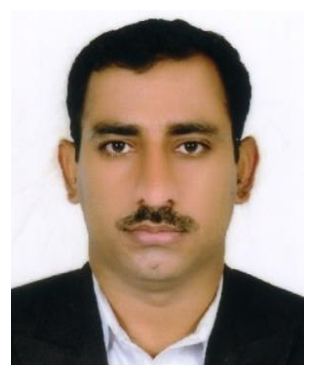

Rasoul Damani (iD SC P received the B.Sc, M.Sc and Ph.D degrees from Sharif University of Technology (SUT), Tehran, Iran in 1998, 2000 and 2015 respectively, all in electrical engineering. He is currently an assistant professor at Chabahar maritime university, Chabahar, Iran. His research interests include the areas of optical communication and underwater communication systems. He can be contacted at email: damani@cmu.ac.ir. 